\title{
Erster Teil, drittes Buch: Der Mensch und sein Selbst oder Metaethik und Übergang
}

\author{
Martin Brasser
}

In diesem dritten Buch des ersten Teils ${ }^{116}$ behandelt Rosenzweig die Frage: was ist der Mensch? Seine Antwort lautet: der Mensch ist in seinem Wesen metaethisch. Ausgangspunkt ist die Feststellung, dass der Mensch ein in sich geteiltes Wesen hat. Er besteht aus zwei Elementen oder Bereichen oder Diskursen.

${ }^{116}$ Die Entstehungsgeschichte dieses Teils lässt sich aus Rosenzweigs Briefen recht präzise datieren: am Abend des 13. September 1918 verlässt Rosenzweig die Stellung auf dem Dub in Richtung des nahe gelegenen Skopje (türkisch Üsküb). Auf dem Weg dorthin wird am 14. September 1918 SE I 2 fertig (GB 152). Über Nisch gelangt Rosenzweig nach Belgrad und verbringt die Tage im Lazarett (GB 153). Dort schreibt er bis zum 31. September den SE I 3 fertig und beginnt am 1. Oktober mit dem «Übergang». Parallel dazu entsteht die Abschrift von I 2 und I 3 (GB 155). Am 10. Oktober 1918 ist auch die Abschrift des «Übergangs» erstellt zusammen mit der Abschrift der Hälfte von SE I 3 (GB 167). Mit Brief vom 11. Oktober kann Rosenzweig also seiner Mutter also insgesamt fünf Abschriften ankündigen: «du ... kannst diese fünf in einen gemeinsamen Umschlag legen und auf den Umschlag schreiben: Die Elemente oder das Immerwährende» (GS 612). Am 19. Oktober ist nur die Abschrift von SE I 3 noch nicht per Post an die Mutter verschickt (GS 613).

How to cite this book chapter:

Brasser, M. 2021. Erster Teil, drittes Buch: Der Mensch und sein Selbst oder Metaethik und Übergang. In: Brasser, M., Bojanić, P. and Ciglia, F. P. (eds.) The Star for Beginners: Introductions to the Magnum Opus of Franz Rosenzweig. Pp. 55-68. London: Ubiquity Press. DOI: https://doi.org/10.5334/bco.f. License: CC-BY 
Auf der einen Seite gibt es den Bereich, in dem alles vorkommt, auf das man sich abstützen und verlassen kann - ein Bereich des Stabilen. Auf der anderen Seite gibt es einen Bereich, von dem aus auf das Stabile im Menschen geblickt wird. Das ist der Bereich, den Rosenzweig die Freiheit nennt. Beide Bereiche bestehen zusammen. Der eine kann den anderen nicht ersetzen. Damit nimmt Rosenzweig den Gedanken auf, den wir in den vorausgehenden Kapiteln schon gelesen haben: den Gedanken von der inneren Zerbrechung, der inneren Differenz. Er wird im dritten Buch des ersten Kapitels wieder aufgegriffen und anthropologisch vertieft.

Um den Kern und die Pointe dieser These zu erfassen, braucht es ein Verständnis für das methodische Vorgehen von Rosenzeig und für seine inhaltliche Argumentationsfolge.

\section{Das methodische Vorgehen}

Methodisch greift Rosenzweig wie bei der Analyse der Elemente Gott und Welt auf eine mathematische Symbolsprache zurück. Sie soll - wie bei Gott und Welt auch - das beschreiben, was der Untersuchungsgegenstand eigentlich ist: «das wahre Sein des Menschen» (55). Die formale Struktur des verwendeten Symbols ist am Aussprechen des Wortes «Ja» formalisiert, das für Rosenzweig im Aussprechen von jedem anderen Wort mit ausgesprochen ist und so dessen Grundlage darstellt. «Ja sagen» heisst mindestens: die Relation zwischen dem, was man sagt, und dem Umstand, dass es gesagt ist, als bestehend bestätigen - und damit eine Relation herstellen, die formal mithilfe der Buchstaben A oder B und einem Gleichheitszeichen ausschreibt. Gott wird in der Gleichung « $\mathrm{A}=\mathrm{A} »$, Welt in der Gleichung « $\mathrm{B}=\mathrm{A}$ » und der Mensch in der Gleichung $« \mathrm{~B}=\mathrm{B} »$ dargestellt. Diese Formalisierung ist lediglich eine Hilfskonstruktion, die in dem Moment wieder verworfen wird, in dem sie ihren Dienst geleistet hat. Sie kommt im zweiten Teil des SE nicht mehr vor. Sie dient nur dazu, das «Sein» oder "Wesen» des Menschen zu erfassen und tut dabei so, als liesse es sich in eine allgemeingültige Formel fassen. Dem ist nicht so. Aber in dem Stadium, in dem sich die Gesamtargumentation des SE jetzt gegen Ende des ersten Teils befindet, ist diese Formel noch hilfreich. ${ }^{117}$ Sie ist abstrakt formal genug, um klarzumachen, dass das «Sein» oder «Wesen» des Menschen nicht angemessen erfasst wird, wenn man sie in allgemeingültige Sachbegriffe zu bringen versucht. Der Anspruch daran ist eigentlich nicht deshalb verfehlt, weil man Sachbegriffe statt formaler Termini zu nutzen versucht, sondern weil man für die Begriffe den Anspruch von Allgemeingültigkeit erhebt, den sie als philosophische Begriffe traditionell haben aber gleichwohl nicht mehr erfüllen können. Im Verlauf des Kapitels wird Rosenzweig deshalb seine Argumentation schrittweise so aufbauen, dass der eigentliche Begriff für das

${ }^{117}$ Die Symbolsprache «B=B» für den Menschen wird ausführlich rekonstruiert bei Samuelson, User's Guide 66ff. 
«Sein» oder «Wesen» des Menschen maximale Anschlussfähigkeit an die nichtphilosophische biblische Sprache hat: der Mensch ist in seinem wahren Kern nicht Selbst, sondern Seele.

Bevor dieser Denkschritt nachvollziehbar ist, braucht es noch das Verständnis für einen methodisch noch davorliegenden Schritt: Rosenzweig bestimmt das «Sein» oder «Wesen» des Menschen wie sein expliziter Gewährsmann Kant und wie sein impliziter Gesprächspartner Hegel transzententalphilosophisch. «Transzendental» heisst hier zunächst das, was als Bedingung dafür angenommen werden muss, dass Menschen Menschen, also sie selbst sein können. Und es heisst zugleich und damit einhergehend, dass dieses «Sein» oder «Wesen» so verfasst ist, dass der Mensch in de Lage ist, über sich selbst hinauszugehen. Dabei denkt Rosenzweit vom Ende seiner Systematik her, d.h. von seinem radikal theo-zentrischen Verständnis des «Seins» und «Wesens» des Menschen, dem dieses «Sein» oder «Wesen» solange nicht zugänglich ist, als es nicht von Gott selbst eröffnet worden ist. Auch diese Verschlossenheit ist Teil des Wesens des Menschen - und eine Methodik, die das Element des Gottesbezugs aus der Erfassung des «Seins» oder «Wesens» des Menschen ausblendet, kommt bis zu genau diesem Punkt der Erkenntnis: der Mensch ist in seinem «Sein» und «Wesen» nur er selbst. Damit ist der im Kern dreistufige Argumentationsschritt Rosenzweig in diesem Kapitel schon genannt: das «Sein» oder "Wesen» des Menschen ist sein Menschsein («Adam»), das aus philosophischer Sicht ein «Selbst» ist, wobei der Kern dieses Selbst «die Seele» ist. Es gehört zu den Grundüberzeugungen Rosenzweigs, dass die Philosophie diese eigene Begrenztheit ihres Blicks auf «Sein» und «Wesen» des Menschen selbst nicht in den Blick bekommt und folglich der Kernbestand des Selbstkonzepts, also die «Seele» des Selbst, selber gar nicht auf den Begriff gebracht hat. Dafür muss man auf die Literatur ausweichen, die zwar nahe bei der Philosophie ist, aber näher noch bei dem Kern der Philosophie, für den die Philosophie selbst blind ist.

Wenn Literatur der Ort ist, an dem vor-theozentrisch «Sein» und «Wesen» des Menschen verständlich und gewissermassen vor-begrifflich richtig verstanden werden, dann ist auch klar, dass dieses «Sein» und «Wesen» nichts Abstraktes ist, sondern Gegenstand von erlebbarer Erfahrung. Literatur begreift sie, wenn sie Menschliches wie Geburt, Tod, Liebe auf der Bühne oder zwischen Buchdeckeln evoziert. So wie Geburt, Tod oder Liebe Erfahrungen der empirischen Welt sind, so sind sie die Erfahrungen des realen «Seins» und «Wesens» dessen, was uns als Menschen ausmacht. Der Schnitt läuft für Rosenzweig nicht zwischen Erfahrung und Transzendentalität - was wäre das für eine Transzendentalität, die nicht zur Erfahrung werden könnte -, sondern zwischen zwei Formen von Erfahrung: diejenige, in der wir uns als Menschen mit anderen verbunden und in Austausch befindlich erfahren, und derjenigen, die uns auf uns selbst und unsere individuelle Eigenart und Unbezüglichkeit zurückwirft. Welche davon die anthropologisch-transzendental argumentativ relevante ist, dürfte aus dem Bisherigen klar geworden sein. Rosenzweig baut seine Argumentation so auf, dass er dabei zeigt: solange das «Sein» und «Wesen» 
des Menschen als «Selbst» konzipiert wird (und das tut alle Philosophie), hat man erst den Anteil an diesem «Sein» und «Wesen» erfasst, der im Vorfeld der «wahren» Philosophie angesiedelt ist, die ihrerseits nur als Theologie möglich ist. Denn nur sie erfasst das «Sein» und das «Wesen» des Menschen als solchen, d.h. als in Beziehung stehenden.

\section{Der Argumentationsverlauf}

Argumentativer Ausgangspunkt des dritten Buchs im ersten Teil des SE ist die These, dass der Mensch eine innere Differenz ist. Dieser Gedanke erinnert an Descartes und dessen Unterscheidung zwischen «res cogitans» und «res extensa» und an Kants Unterscheidung zwischen der Welt der Erscheinung und der Welt der Freiheit. Die Frage, die sich deshalb sogleich stellt, lautet: Wie ist die Einheit beider Teile beschaffen. Hier sieht Rosenzweig anders als Descartes oder Kant eine Steigerung in der Art, wie diese Einheit beschaffen sein kann. Diese Steigerung zeigt Rosenzweig in diesem Kapitel dadurch an, dass er zunächst vom Menschen als solchem spricht, dann vom Menschen als einem Selbst und im dritten Schritt vom Menschen als Seele. Der Übergang vom Begriff Mensch zum Selbst ist eine Konsequenz aus der Begriffsanalyse, die Rosenzweig im vorliegenden dritten Buch des ersten Teils vornimmt. Der Übergang vom Selbst auf die Seele kann, so Rosenzweig, nicht mehr durch eine Analyse des Begriffs «Seele» erfolgen. Sondern dieser Übergang braucht das, was Rosenzweig am Ende des Kapitels «die Umkehr» nennt. Hier beginnt das Ende des philosophischen Räsonnierens und der Einstieg in das, was Rosenzweig denkerisch an Neuem bringen in die Philosophie einführen möchte. ${ }^{118}$

Es geht um das, was Kant so ausgedrückt hat: dem Glauben Platz machen. Kant ist dann auch der erste Bezugspunkt, mit dessen Hilfe Rosenzweig in seine Argumentation einsteigt. Kant ist der Gewährsmann, auf den sich Rosenzweig für seine eigene Analyse vom Wesen des Menschen beruft, und zwar sowohl in Bezug auf dessen Erkenntnistheorie, auf die sich Rosenzweig in diesem Kapitel explizit bezieht, als auch auf dem Feld der Moralphilosophie. Mit Kant teilt Rosenzweig die Vorstellung darüber, wohin die Begriffsanalyse weisen soll: in den Glauben hinein. Dort angekommen, wird - so ist das Versprechen von 52 - wir das nicht-glaubende Wissen in eine ganz einfache und klare Erkenntnis münden. Worin diese Erkenntnis besteht, das wir dann in den späteren Kapitel dargelegt.

Am Anfang dieses Wegs steht eine Verwirrung (54): Wo soll man anfangen, wenn man sich fragt "was ist der Mensch»? Man weiss nicht, wo genau

${ }^{118}$ Im Brief vom 1. Oktober 1918 schreibt Rosenzweig an Gritli seine Zusammenfassung des dritten Buches. Er hat «eben I 3 zu Ende geschrieben und es gefällt mir ganz gut» (GB 155). In seiner Zusammenfassung stellt Rosenzweig insbesondere den Bezug zum «Rudibrief», der sog. Urzelle des Sterns heraus. 
man anfangen soll. Man hat, so Rosenzweig, zunächst nichts in der Hand, von woaus man anfangen könnte. Und genau dieses «Nichts» macht Rosenzweig zum Ausgangspunkt seiner Überlegungen, wie das zum ersten Mal schon bei «Gott», dann zum zweiten Mal bei der «Welt» der Fall gewesen war.

Diese Überlegungen sind also methodisch geführt, und zwar in einem Dreischritt: ausgehend von einem bestehenden Faktum, das schon allein dadurch, dass es besteht, eine komplexe Welt eröffnet, wenn man das, was da besteht, analysiert («schaffendes Ja»). Man muss nur gedanklich analytisch genug vorgehen, um nachvollziehen zu können, was gedanklich-analytisch alles in diesem Ausgangspunkt implizit bereits mitgegeben ist. Dann entsteht gewissermassen begriffslogisch-automatisch der nächste Schritt: man sieht das, was verneint worden ist. Auch hier öffnet sich erneut eine komplexe Welt («das zeugende Nein»). Und schliesslich braucht es den Schritt, der die Verbindung zwischen den Ergebnissen des ersten und denen des zweiten Schritts herstellt («das gestaltende Und»).

Diese Gedankenbewegung nimmt ihren Reiz und ihre Überzeugungskraft daraus, dass man mitvollzieht, wie konsequent der eine Schritt aus dem anderen folgt. Daran hängt die Plausibilität der Argumentation, die sich einer Hegel'schen Dialektik bedient, ausgehend von einem negativen Befund, dem «Nichts des Menschen».

Als Ausgangspunkt nimmt Rosenzweig zunächst das, was der gesunde Menschenverstand antwortet, wenn er fragt: was unterscheidet den Menschen von Gott und was unterscheidet ihn von der Welt? Die Antwort auf diese Fragen ergibt eine Antwort auf die Frage nach dem Wesen des Menschen, wenn die jeweiligen Antworten miteinander kombiniert werden. Gott ist - gemäss Rosenzweig - immer «dieser Gott», also individuell. Und die Welt ist ein Wechsel von Entstehen und Vergehen. Die Kombination aus beiden Aspekten ergibt das Wesen des Menschen: der Mensch lebt den Wechsel von Entstehen und Vergehen als Individuum. Das heisst: genau einmal. «Vergänglichkeit ist sein Wesen». Zu dieser Vergänglichkeit kommt nach Rosenzweig ein unbestreitbares Fakt: die Fähigkeit des Menschen, sich auf diese individuelle Vergänglichkeit zu beziehen.

Von der Art, wie Rosenzweig genau diesen Bezug beschreibt, hängt der ganze weitere Weg der Analyse ab. Dieser Bezug ist rein nur auf das je eigene Individuelle gerichtet. Man sieht die ganze Welt jeweils nur aus der individuell-eigenen Optik, geradezu - sit venia verbo - «autistisch». Rosenzweig bezeichnet den Menschen auf dieser Stufe als «einzelnes und doch alles». Dieser Bezug ist ausserdem darauf aus, sich selbst auf Dauer zu stellen. Am besten wäre hier die ungekürzte Dauer, also die Unendlichkeit. Kürzere Dauer haben die Eigenschaften, die den individuellen Menschen Zeit seines Lebens prägen. Diese Eigenschaften machen das am Menschen aus, was Rosenzweig «den Charakter» nennt. Damit ist bereits in einem ersten und einfachen Denkschritt eingelöst, was eingangs festgestellt worden war: der Mensch ist ein geteiltes Wesen. Wir wissen jetzt besser, worin dieser Teil seines Wesens besteht. Die Teile selbst sind 
geklärt: es gibt das, was man ein «Nichts» bezeichnen kann, und das «Feste» im Menschen, das Rosenzweig dessen «Charakter» nennt.

Rosenzweig liebt es, in der geometrischen Figur des Sterns zu denken. ${ }^{119}$ Mit dem bisherigen Gedankengang ist ein Teil der noch zu erarbeitenden Formel für das Wesen des Menschen bereits gefunden. Die Eigenheit des Charakters des Menschen kann mit einem einfachen «B» bezeichnet werden. Der Sinn dieser Operation erschliesst sich eigentlich erst, wenn man am Schluss steht und die ganze Formel vor sich hat. An dieser Stelle wird zunächst einfach eingeführt: der Charakter des Menschen ist ein einfaches «B».

Methodisch folgt jetzt der zweite Schritt, der des «zeugenden Nein». Man muss dafür das erreichte Ergebnis seinerseits verneinen bzw. genauer: das verneinte erreichte Ergebnis als Teil des Ganzen ansetzen, das eben der Mensch ist. Und was ist das im Menschen, das den Charakter verneint? Das ist, so Rosenzweig, der freie Wille. Das Faktum der freien Stellungnahme gehört genauso zum Wesen des Menschen wie die Tatsache, dass er oder sie einen ganz spezifischen Charakter hat. Dieser Wille selber ist endlich, weil er nicht so allmächtig ist wie bei Gott. Als Symbol für den freien Willen benutzt Rosenzweig wieder ein «B». Und weil der Wille etwas ist, was sich auf etwas bezieht («ich will etwas»), kommt noch ein Beziehungszeichen hinzu: den Teil des Menschen, der seinen Willen ausdrücken soll und seinem Willen entspricht, schreibt Rosenzweig als « $\mathrm{B}=»$.

Damit hat Rosenzweig die beiden zentralen Teile oder Elemente am Menschen bestimmt: seinen Charakter und seine Freiheit.

Man kann das als eine freie Verdichtung der Gedanken ansehen, die schon Kant zur Frage nach dem Menschen vorgetragen hat. Rosenzweig führt deshalb hier mit guten Grund Kants Name ein - einmal mehr. Bei der nächsten Frage aber kann Kant nicht mehr weiterhelfen. Sie lautet: wie hängen beide Teile des Menschen zusammen? Hier geht Rosenzweig über Kant hinaus. Sofern der Mensch als die Einheit aus Charakter und Wille verstanden wird, nennt Rosenzweig ihn «das Selbst». Das Selbst ist sozusagen das «Und» aus dem ersten «Ja» und dem anschliessenden «Nein». Dass es eine solche Einheit gibt, ist wenig strittig. Aber wie sieht diese aus? Die Antwort auf diese Frage entfaltet Rosenzweig im Folgenden. Er geht dabei von einer Beobachtung aus, die beim Willen ansetzt. Rosenzweig beobachtet einen Widerspruch im Willen selbst. Dieser Widerspruch wird deutlich, wenn man sich vor Augen hält, dass Freiheit etwas ist, das ausdrücken soll, dass man an nichts gebunden ist. Freiheit heisst, «causa sui et non altrius» zu sein. Als Menschen sind wir aber, wie soeben dargelegt, an den Charakter gebunden. Das ist nun aber nicht nur ein

${ }^{119}$ Am 4. Oktober 1918, also 14 Tage nach dem Beginn der Niederschrift des SE am 21. August 1918 schreibt Rosenzweig an Gritli darüber, dass er im Motiv des Sterns den ganzen Inhalt des zu schreibenden Buches vor Augen hat: "Also ich sah den Stern und merkwürdigerweise drehte er sich um sich selbst und darin war alles was ich noch zu schreiben habe, zu sehn.» (GB 159). 
Problem, entstanden aus dem Gegensatz von Charakter und Wille, wobei der Wille tendentiell das Unendliche will, es aber nicht (erreichen) kann aufgrund der Tatsache, an einen Charakter gebunden zu sein. Sondern - und darin liegt Rosenzweigs spezifische Beobachtung - das ist ein Problem im Willen als solchem. Der Wille ist Ausdruck dessen, dass wir frei sind - und zugleich will jeder Wille als Wille sich binden. Er will etwas. Dieser Widerspruch trägt jeder Mensch als solcher in sich aus und zwar nicht erst dann, wenn er seinen Charakter bejaht oder verneint. Sondern überhaupt bereits dann, wenn er überhaupt einen Willen hat. Sofern und weil der Wille diese doppelgleisige Tendenz hat und somit nie wirklich befriedigt werden kann, nennt Rosenzweig ihn den «Trotz». Der Trotz ist «des freien Willen Gestalt».

Die gesuchte Einheit, das Selbst, ist also näher betrachtet «ein Und aus Trotz und Charakter».

Noch einmal betont Rosenzweig - und an der regelmässigen Wiederholung dieses Gedankens erkennt man, wie wichtig dieser Gedanke für die Argumentation Rosenzweigs ist: dieses Selbst hat «keine Beziehung zu den Menschenkindern, immer nur zu einem einzigen Menschen, eben dem Selbst». Dieses Selbst ist «Adam, der Mensch selbst».

Um diese Unbezogenheit des Selbst klarer zu machen, grenzt Rosenzweig diese Unbezogenheit, die sich gewissermassen nach oben richtet, gegen die Unbezogenheit ab, bei der das, was man selbst ist, aus dem Bezug zu Anderen gewonnen wird. Rosenzweig nennt dies «die Persönlichkeit». Und zusätzlich grenzt Rosenzweig das Selbst sozusagen nach unten ab, gegen die Individualität, d.h. gegen das blosse Vorhandensein des numerisch Einzelnen. Als solches Selbst ist der Mensch eine Bezogenheit, wenn man es formelhaft ausdrücken will «ein =», aber als solche eben unbezogen, d.h. "reine Insichgeschlossenheit, bei der sich der Mensch nur auf sich selber bezieht». Die Formel des Selbst lautet folglich $« B=B »$. Das ist ganz und gar nichts Negatives. Es ist vielmehr der Ausdruck für die besondere Nähe des Menschen zu Gott. Rosenzweig verstärkt hier noch einmal die Gleichsetzung von Selbst mit Adam und bezeichnet das dergestalt autonome Ich als «Ebenbild Gottes». Also nicht die Bezogenheit, sondern die Unbezüglichkeit und damit die Gabe, bei sich selbst zu sein und zu bleiben, ist das, was den Menschen so sein lässt wie Gott. Rosenzweig adelt die Unbezüglichkeit zu einem exklusiven Prädikat menschlicher Würde. Er schliesst sich damit der Auffassung der Aufklärer an, die der Meinung sind, dass es die Autonomie ist, die dem Menschen die Würde gibt.

\section{Eine erste Zwischenüberlegung}

Im Grunde ist die oben gestellte Frage jetzt beantwortet. Das begriffliche Gerüst ist aufgebaut. Was jetzt noch folgt, sind Konsequenzen und Plausibilisierungen dessen, was auf rein begriffslogischer Ebene bisher bereits über das Wesen des Menschen gesagt worden ist. Rosenzweig zieht im Folgenden zwei 
Konsequenzen in 62 - 64. Dann blickt er auf die Kulturgeschichte Indiens (6566), Chinas (67-68), des Vorderen Orients (69) und des Alten Griechenlands (70-71). In letzterem erkennt Rosenzweig die Figur des Selbst als tragendes kulturelles Identifikationsmuster. Schliesslich versucht er durch eine kunsttheoretische Überlegung seine Idee des Selbst weiter plausibel zu machen. Damit könnte die Argumentation an ihr Ende gelangt sein - und sie wäre es auch, wenn da nicht ein Thema oder Problem noch offen geblieben wäre: Der Mensch als Selbst kommt ohne Beziehung nach aussen aus. Der reale, konkrete Mensch aber tut das nicht. Folglich ist das Selbst noch nicht die Beschreibung des tatsächlichen Menschen. Mehr noch: wenn man nur nach dem Ebenbild Gottes leben würde, könnte man immer noch «alles Fremde allein dort im Eigenen und als Eigenes erblicken». Das wiederum wäre der Autismus, den kein Mensch jemals wollen kann. Und zwar gerade auch als Ebenbild Gottes nicht. Wie entsteht bei Wahrung der Autonomie Bezogenheit? Das zu zeigen muss der nächste Denkschritt sein, wenn man die Frage nach dem Menschen so beantworten will, dass man nicht nur einer kohärenten Begriffsphantasie folgt, sondern die passende Beschreibung dessen vor Augen geführt bekommt, was Menschen wirklich sind. Genau und erst dann ist Philosophie im Rosenzweig'schen Sinn empirisch. Und dabei gilt, wenn die bisherige Beschreibung passend war und das Beste des Menschen tatsächlich darin besteht, ein Selbst zu sein: aus dem Menschen selbst kann diese Bezogenheit nicht kommen. Der Schritt in die eingangs bereits angesprochene Dimension des Glaubens wird an dieser Stelle unausweichlich. Auf diese Spitze und «Kehre» läuft Rosenzweigs Argumentation letztlich zu. Bevor diese Wende vollzogen wird, benennt Rosenzweig zwei Konsequenzen aus seiner Argumentation.

Die erste Konsequenz betrifft die Frage, wann das, was bisher über das Selbst rein argumentativ-begrifflich gesagt wurde, auch tatsächlich erlebt und erfahren wird. Rosenzweigs Antwort lautet. Zum einen kann dies ab dem Moment eintreten, in dem ein Mensch erstmals seine eigene individuelle Sexualität erlebt. Und zum anderen ganz besonders in dem Moment, in dem ein Mensch sein Selbst gerade endgültig verloren hat - «auf dem erstarrten Antlitz eines Toten».

Die zweite Konsequenz betrifft die Frage, welche Rolle dann noch allgemeine sittliche Verpflichtungen spielen, wenn das Selbst so sehr auf Autonomie und Selbstbezogenheit abgestellt ist. Hier kann man nach allem Bisherigen nicht anders antworten als so: das Selbst steht über der objektiven sittlichen Welt. Es ist metaethisch.

Für die kulturgeschichtliche Plausibilisierung der These vom Selbst «in seiner gebirgshaft edel stummen Einsamkeit» greift Rosenzweig im Wesentlichen auf seine auch in anderen Stellen des Sterns vorgenommene Dreigliederung der Varianten von Möglichkeiten zurück: Indien - China - Griechenland. Die jeweils best gelungene Variante findet sich immer im Alten Griechenland. Indien und China sind jeweils noch defizitärere Varianten, die je eine der beiden Bestandteile - hier des Selbst - übergewichten und zueinander komplementär sind, während das alte Griechenland die Synthese aus den beiden 
komplementären Polen bereits herstellt, aber noch nicht in der gelungenen Form. Vollgestalt des Selbst ist der Held in den Tragödien der attischen Dichtung. Dort ist das Selbst sozusagen literarische Gestalt geworden. In Indien war dies nicht möglich. In der dortigen Kultur liegt der Akzent darauf, nur die Seite des Charakters zu betonen. So jedenfalls deutet Rosenzweig die Tatsache, dass die indische Kultur stark vom Kastenwesen geprägt ist. Sogar die buddhistische Religionsvariante in Indien konnte, so Rosenzweig, daran nichts Wesentliches ändern, sofern auch der Buddhismus dort von der Idee geprägt ist, einen bestimmten Charakter, nämlich den der Erlöstheit, zu erreichen. China dient hier als Kontrastfolie: Konfuzius, Litaipe, Laotse - sie alle wollen letztlich die Reduzierung des Charakters bis zu dessen vollständiger Auflösung, die letztlich nur der vollkommene Mensch erreicht. Was hier idealtypisch übrig bleibt, ist ein Nicht-Ich, das im Rosenzweig'schen Konzept von Anthropologie der reine Wille ist. Die Einheit beider Seiten gibt es erst in der Figur des tragischen Helden der antiken attischen Tragödien.

Diese Figur ist schon in der Bibel in den Gestalten von Simson oder Saul und im Vorderen Orient in der Gestalt des Gilgamesch vorentworfen. ${ }^{120}$ Die Attischen Dichter, allen voran Aischylos, bringen sie dann in die europäische Kulturgeschichte ein. Wieso aber ist gerade dieser Held der Inbegriff des Selbst und woran erkennt man das? Rosenzeig bringt hier mehrere Beobachtungen bei. Er führt zugleich und en passant einen zentralen Gedanken ein. Die Selbstabgeschlossenheit der Helden der antiken Tragödien ersieht man daran, dass die Helden der attischen Tragödien nie selber Dialoge führen. Das tut gemäss der Dramaturgie jeweils der Chor. Ein Ausstieg aus der Selbstabgeschlossenheit ist zudem in keiner der attischen Tragödien jemals vorgesehen: es gibt keine Liebesszenen. Im Showdown der Tragödie wird die Selbstabgeschlossenheit vom Helden selbst jeweils ausdrücklich so gewollt. Der Grundsatz lautet hier: Ausdruck der Bezugslosigkeit ist die Sprachlosigkeit. Dabei will der antike Held doch nur das, was jedes andere Selbst auch will: bleiben, Bestand haben und letztlich unsterblich sein.

Unsterblichkeit meint aber in der Sache durchaus mehr als nur dies, dass man für unbegrenzte Zeit fortbesteht. Was genau sie bedeutet, wird von Rosenzweig an dieser Stelle seiner Argumentation noch nicht gesagt, sondern nur angedeutet, wenn er von «Unsterblichkeit im neuen Sinn» spricht. Man ahnt es freilich schon: es geht um eine Unsterblichkeit, die nicht nur unendliches Weiterleben meint, sondern die zugleich frei ist von der entscheidenden Begrenztheit, die das Selbst noch in sich trägt - von der Sprachlosigkeit.

Tatsächlich aber hat die antike Philosophie keinen derartigen qualitativen Begriff von Unsterblichkeit entwickelt. Sie ist, so Rosenzweig, der Idee verhaftet geblieben, dass es etwas geben könnte, das das Ich dauerhaft trägt so wie das Wasser die Schiffe oder die Wurzel den Baum trägt. In der antiken Philosophie heisst dieser Träger «Seele». Aber in diesem Denken ist die «Seele» dann

${ }^{120} \mathrm{Zu}$ den Vorgestalten ausführlicher vgl. Samuelson, User's Guide 70-76. 
doch wieder reduziert auf einen Teil des Menschen und beschreibt nicht sein ganzes «Selbst».

\section{Eine zweite Zwischenüberlegung}

Damit liegen alle argumentativen Fäden bereit, um die Idee des Selbst in die der Seele zu überführen. Man ahnt es als Leser: die Seele ist das Selbst, das durch Sprache in die Welt der Beziehung eingeführt wird. Als solches ist das Selbst «Seele» in einem nicht mehr antik-griechischen, sondern in einem der Bibel entnommenen Sinn.

Bevor aber nun der Schritt aus der Welt der Philosophie in die Welt der Theologie, aus der Welt des Heidentums in die Welt der Bibel gemacht wird, gibt es einen weiteren Versuch, das begriffslogische Gerüst des Selbst anschaulich zu machen. Dem kulturgeschichtlichen folgt der kunsttheoretische Zugang. Dadurch wird auch präzisiert, welche Sprache Rosenzweig im Sinn hat, wenn er Sprache als Mittel der Befreiung aus der Autonomie verstanden wissen will. Es ist die Sprache des gesprochenen, des akustisch laut gewordenen Wortes. Die Kunst ist die Sprache, der es gelingt, das Selbst anzusprechen, ohne dafür Worte verwenden zu müssen. Kunst ist so stumm wie der antike Held und spricht genauso wie er: durch ihre Wirkung, die ohne Worte wirkt. Kunst wirkt wie das stumme Selbst und sie bewirkt in den Menschen, auf die sie wirkt, dass sie selber stumm bleiben. Kunst «geschieht von Selbst zu Selbst, von einem Schweigen zum anderen Schweigen.» Aber so ist die Welt noch nicht die Welt, wie sie ist, wenn sie erfüllt und bewohnt wird nicht nur vom Selbst, sondern vom konkreten Menschen. Wie das Selbst aufwacht zur Bezogenheit, wie die Sprache so wirkt, dass sie sprechend macht, wie Worte die Selbstbezogenheit in Bezogenheit auf den Anderen macht, davon handelt der zweite Teil des Sterns der Erlösung. Um das zu verstehen, verlassen wir zugleich die Welt Chinas, Indiens, Griechenlands und allen Heidentums und gehen über die Welt des jüdisch-christlichen Glaubens.

\section{Übergang}

Es folgt das letzte Kapitel des ersten Teils des SE mit dem Titel „Übergang“. Damit ist dessen Funktion bereits angezeigt: dieses Kapitel verbindet die bisherigen Argumentationen über Gott, Welt und Mensch mit denen des zweiten Teils des SE. Dies tut Rosenzweig dadurch, dass er Bewegung in die Zuordnung der drei Elemente zueinander bringt. Sie stehen nicht isoliert jedes für sich, sondern jeweils für eine Epoche in der Weltgeschichte: für die Vergangenheit steht die entwickelte Gottesvorstellung, für die Gegenwart das Konzept des Menschenbildes und für die Zukunft das, was als Element „Welt“ erarbeitet worden ist. Diese Dynamisierung und Historisierung der Ergebnisse macht diese noch anschlussfähiger an das, was in den folgenden Teilen des SE weiter entwickelt werden wird. Dort entwirft Rosenzweig ein weltgeschichtliches Szenario mit 
Gott an dessen Anfang, dem Menschen in dessen Mitte und der (erlösten) Welt als einer in der Zukunft liegenden Zielgestalt. Wenn die bisherigen Elemente nicht nur einzeln nebeneinander liegende Abstrakta, sondern in einem zeitlich spezifizierten Verhältnis zueinander stehen, dann sind sie noch mehr das, was sie immer schon sein sollten bzw. eigentlich auch immer schon sind: die heidnische Vorwelt dessen, was im Kern und in Wahrheit die Welt der Theozentik ist. Wäre diese Vorwelt ganz und gar über sich selbst aufgeklärt, dann wüsste diese Welt auch das. Aber sie weiss ja nicht einmal, dass die drei Elemente in einem geordneten Verhältnis zueinander stehen. Das zu zeigen, ist Aufgabe des Abschnittes „Übergang“.

Dies nicht zu wissen, ist nicht Schuld oder moralische Verfehlung, sondern liegt gewissermassen in der Natur der Elemente. Im Ergebnis der bisherigen Argumentation stehen sie einfach nebeneinander. Sie sind aber auch Erklärungskonkurrenten: jedes Element kann als Ursprung jedes anderen Elementes verstanden werden, sobald es als universal gültig genommen wird. Dann aber werden zwei Elemente auf ein drittes reduziert, ohne dass eine der drei dabei möglichen Varianten eine höhere Plausibilität für sich beanspruchen könnte.

Gleichwohl ist es besser, diese drei Elemente in eine Ordnung zu bringen, als sie nebeneinander stehen zu lassen. So legt sich der „Wirbelwind der Widersprüche“ (Se 93). Denkerische Ruhe stellt sich für Rosenzweig ohnehin erst dann ein, wenn Erkennen in die Form eines Systems gebracht worden ist. Dessen Umrisse zeichnet Rosenzweig im Folgenden: a) dieses System erhält seine Ordnung dann, wenn seine Elemente untereinander in eine Beziehung gebracht sind und b) wenn dafür die einzelnen Bestandteile selbst nicht statisch, sondern dynamisch als Prozesse verstanden worden sind, die c) einen Platz in der Geschichte einnehmen.

\section{Ordnung entsteht durch Beziehung}

Ordnung entsteht durch Relation. Ordnung ist dabei das Ergebnis einer interpretatorischen Eindeutigkeit, die nicht aus dem Fakt, sondern aus der Relation, in der das Fakt eingebunden ist, entsteht. Rosenzweig bringt zwei Hinweise: ob die Zahl „3“ eine Vielheit („drei einzelne Dinge“) oder eine Einheit („die Drei“) bedeutet, ersieht man aus der Gleichung, in der die Zahl 3 Verwendung findet: „3 $3=3 \times 1$ “ oder, $3=1 \times 33^{“ 121}$. Das ist der erste Hinweis.

${ }^{121}$ Samuelson, User's Guide 79 weist zurecht auf die Analogie der Fragestellung hin: ist «3» als Einheit oder als Vielheit zu verstehen - sind auch die Elemente als Einheit oder als Vielheit zu verstehen? Die Antike konnte diese Frage nicht beantworten. Wenn «Antike» für das Äusserste steht, was nicht-theozentrische Weltsicht aus sich selbst heraus denkerisch leisten kann, dann wird die Radikalität dieser Beobachtung deutlich - verschärft durch die Beobachtung Rosenzweigs am Kapitelanfang, dass jedes Element «sich monistisch als das Ganze» (SE 92) zu setzen in der Lage wäre. 
Der zweite lautet: Fakt und Ordnung verhalten sich wie Integral zu Differential. Mittels Integralrechnung werden die Grössen von Flächen errechnet und als Fakt ausgegeben. Die Differentialrechnung berechnet die Veränderung von Funktionen, indem es Eingabewerte und Ausgabewerte aufeinander bezieht. Das gilt auch übertragen auf die drei Elemente: erst wenn sie „miteinander in eindeutige, ... wirkliche Beziehung treten" (SE 94), verlieren sie den Schwindel erregenden „Wirbel der Möglichkeiten“ (SE 94). Mehr noch und trotz allem, was bisher denkerisch geleistet worden ist: dann können diese drei Elemente überhaupt „erst erkannt werden“ (SE 94). Dabei sind die drei Elemente in sich selbst durchaus beziehungsfähig, weil selber eigentlich nicht statisch, sondern dynamisch, d.h. sie tragen „in sich selbst ... die Kraft ..., aus welcher Bewegung entspringt" (SE 96).

\section{Die Elemente sind dynamisch}

Die Herleitung dieses Aspekts der Elemente bezieht sich eigentlich nicht so sehr auf die Elemente selbst, sondern auf den Vorgang ihrer Thematisierung in den vorausgehenden Büchern. Als reinen Erkenntnisprozess will Rosenzweig dies jedoch nicht verstanden wissen. Dieser Erkenntnisprozess ist Teil der Elemente selbst und deren Hervortreten als das, was sie in ihrem Sein und Wesen sind. Wenn man einmal eingesehen hat, dass die Elemente tatsächlich so sind, wie sie analysiert worden sind, dann sind sie in ihrer Tatsächlichkeit in Erscheinung getreten. Reflektiert man diesen „ontologischen Erkenntnisprozess", dann kann man - so Rosenzweig - eine Art Verdrehung oder Umkehr der Verhältnisse feststellen. Die Herleitung der drei Elemente startete jeweils beim Nichts des Wissens und war so angelegt, dass dieses Nichts als produktive Grösse letztlich der Ursprung dessen war, was dann als Element in seiner Tatsächlichkeit erkannt und als faktisch (so) existierend postuliert werden konnte. Der Vorgang dieser Postulierung aber dreht diese Sachlogik der Entstehung um (obwohl die Verlaufslogik von vorher-nachher natürlich bestehen bleibt): jetzt wird nicht mehr das Nichts das Bewirkende, sondern das Bewirkte - also jedes Element - als die Ursache von Wirklichem angesehen und auch faktisch so in Kraft gesetzt ${ }^{122}$. Das ist bei Gott und beim Menschen gut nachvollziehbar (weniger gut beim Element Welt). Dabei ist wichtig zu sehen: das Fakt der Reihenfolge von Nichts und Element hat sich nicht verändert, nur die Ordnung, in die Anfang und Ende der Bewegung eingebettet ist: das Verhältnis von Ursprung und Wirklichkeit dreht sich um - der Moment der Umkehr ist

${ }^{122}$ Vgl. Samuelson, User's Guide 81 mit dem Akzent auf dem In-Sich-Sein der Elemente als Ausgangspunkt der Transformation: «... the objects of knowledge become transformed from being something in themselves to being origins for the movement of reality (Wirklichkeit)." 
der Moment, in dem ein Element seinen Status der Tatsächlichkeit erlangt hat. Durch diesen Status kommt sozusagen Bewegung in die Zuordnung - und damit wird das Verhältnis der Elemente zueinander dynamisch. „Aus fertigen Ringen werden Glieder einer Kette." (SE 97). An welchem Ort in dieser Kette sich welches Element näherhin befindet, wird im dritten und letzten Argument, der Historisierung der Elemente, noch kurz skizziert.

\section{Die Historisierung der Elemente}

Für Rosenzweig sind die drei Elemente - Gott als lebendiger Gott des Mythos, die Welt als plastische Welt der Kunst und der Mensch als der Held der Tragödie - Ausdruck von „reife(m) Heidentum“ (SE 97). Das ist in einem doppelten Sinn zu verstehen: geschichtlich-historisch und systematisch. Rosenzweig sieht beide Aspekte zusammen: die griechische Antike ist im Grundcharakter der Wissenschaften von Gott, Welt und Mensch auch heute noch gegenwärtig. Und nun historisiert Rosenzweig noch einen Schritt weiter: sofern schon für die Antike die drei Elemente historisch geordnet waren, sind sie es als systematische Grundlage der Wissenschaften auch heute noch. Die historische Ordnung der drei Elemente sieht so aus: schon für die klassischen Griechen war ein Gott als lebendiger Gott des Mythos geschichtliche Vergangenheit und deren Gegenwart vom Selbstverständnis des tragischen Helden geprägt (so dass für die dritte Zeitdimension Zukunft dann das Element Welt übrig bleibt). Diese historische Ordnung ist auch die systematische: „Gott war von uran, der Mensch ward, die Welt wird.“ (SE 99). Was bisher aus Gründen, also philosophisch entwickelt worden und entstanden („geboren“) ist, ist im Licht dessen, was jenseits der Philosophie gewusst wird, eine „Schöpfung“ - und damit lüftet Rosenzweig am Ende des Übergangs schon ein ganz klein wenig den Schleier und gibt den Blick in den Bereich jenseits der Philosophie frei, indem er die Begriffe einführt, die ab jetzt leitend sein werden: Schöpfung und Offenbarung. Der „Übergang des Geheimnisses in das Wunder“ (99) ist denkerisch-argumentativ vollzogen. Denn „Schöpfung“ in einem vor-theozentrischen Sinn ist bisher bereits demonstriert worden: es ist gezeigt worden, wie die Elemente aus ihren Nichts heraus entstehen - und genau darin lag auch (die Beschreibung des Prozesses) ihr(es) Offenbarwerden(s). Die Elemente sind nun als solche für das Erkennen offenbar. Das dritte Stichwort, das in der nun folgenden theo-zentrischen Argumentation der Teile 2 und 3 des SE eine gleich zentrale Rolle spielt, ist die Erlösung. Sie kann hier noch nicht erwähnt werden, weil sie nicht aus den Elementen heraus, auch nicht aus der Perspektive der umgekehrten Elemente heraus, zugänglich gemacht werden kann. Dafür wird Rosenzweig eine ganz eigene Methodologie entwickeln, deren Grundlagen in der gesprochenen Sprache Rosenzweig erst im zweiten Teil des SE legen wird. 


\section{Weiterführende Lektüre}

Freund, E.-R., (1959), Die Existenzphilosophie Franz Rosenzweigs. Ein Beitrag zur Analyse seines Werkes „Der Stern der Erlösung“, 2. durchgesehene Auflage, Hamburg, Meiner.

Santner, E., (2010), Zur Psychotheologie des Alltagslebens. Betrachtungen zu Freud und Rosenzweig, Zürich, Diaphanes. 